\title{
Quenched invariance principle for random walks on dynamically averaging random conductances*
}

\author{
Stein Andreas Bethuelsen ${ }^{\dagger} \quad$ Christian Hirsch ${ }^{\ddagger \S} \quad$ Christian Mönch"
}

\begin{abstract}
We prove a quenched invariance principle for continuous-time random walks in a dynamically averaging environment on $\mathbb{Z}$. In the beginning, the conductances may fluctuate substantially, but we assume that as time proceeds, the fluctuations decrease according to a typical diffusive scaling and eventually approach constant unit conductances. The proof relies on a coupling with the standard continuous time simple random walk.
\end{abstract}

Keywords: random walk; dynamic random environment; invariance principle. MSC2020 subject classifications: 60K35; 60F10; 82C22.

Submitted to ECP on September 29, 2020, final version accepted on November 4, 2021. Supersedes arXiv: 2009. 10927.

\section{Introduction}

Random walks in random environments were first introduced in [18] and have since evolved into a flourishing field of research with manifold connections to other branches of probability, physics and chemistry. In this note, we focus on random walks on dynamic random conductances, which form a rich class of models within this framework. Here, the walker moves according to jump rates on the edges of a given base graph which themselves are evolving according to some stochastic process simultaneously with the movement of the walker. We refer the reader to [9] for an excellent overview of the plethora of models studied in the literature and to [1,8] on the topic of invariance principles.

Despite the variety of different models considered to date, they almost exclusively do not deviate from one central assumption: the environment should be time-stationary.

*The authors acknowledge support from the DFG through Scientific Network Stochastic Processes on Evolving Networks". The research of CM is funded by the Deutsche Forschungsgemeinschaft (DFG, German Research Foundation)-443916008. The authors would like to acknowledge the financial support of the CogniGron research center and the Ubbo Emmius Funds (Univ. of Groningen).

${ }^{\dagger}$ University of Bergen, Department of Mathematics, Allegaten 41, 5020 Bergen, Norway. E-mail: stein. bethuelsen@uib.no

${ }^{\ddagger}$ Aarhus University, Department of Mathematics, Ny Munkegade, 118, 8000, Aarhus C, Denmark. Email: hirsch@math.au.dk

${ }^{\S}$ Bernoulli Institute for Mathematics, Computer Science and Artificial Intelligence, University of Groningen (Univ Groningen), Nijenborgh 9, NL-9747 AG Groningen, Netherlands.

"CogniGron (Groningen Cognitive Systems and Materials Center), University of Groningen (Univ Groningen), Nijenborgh 4, NL-9747 AG Groningen, Netherlands.

"Johannes Gutenberg-Universität Mainz, Institut für Mathematik, Staudingerweg 9, 55099 Mainz, Germany. E-mail: cmoench@uni-mainz.de 
Random walks on dynamically averaging random conductances

Results outside this scope are rare and require either rather strong mixing assumptions $[3,16]$ or depend on model specific features $[7,11]$, or prove results weaker than a full invariance principle, such as laws of large numbers $[4,6]$.

However, the time-stationary setting completely ignores a very natural form of dynamics, namely those converging to a common deterministic limiting value with decaying fluctuations over time. For a simple example, we may think of the timeaverages of renewal processes attached to each of the edges. Moreover, since the law of large numbers is so ubiquitous, even far more elaborate models such as KPZ-type interface growth processes are of this nature [12, 15].

We show that when considering such a non-stationary time-averaging setting on $\mathbb{Z}$ with nearest-neighbor bonds, then a quenched invariance principle holds under surprisingly general conditions. Our central requirement is sufficiently strong polynomial decay of the probability that the environment fluctuates super-diffusively over time. In particular, we work without any kind of mixing condition - be it in space or time. As a specific example, we show that environments based on renewal processes fit into this setting. The proof of the invariance principle relies on a coupling construction to a simple random walk that crucially exploits the one-dimensional structure of the underlying graph.

In Section 2, we introduce precisely the super-diffusive concentration condition and state the invariance principle, which is then proved in Section 3.

\section{Model and quenched invariance principle}

Consider the integer lattice $\mathbb{Z}=(V, E)$ with edges drawn between successive sites and let $\left\{\Lambda_{e}(\cdot)\right\}_{e \in E}$ be a family of almost surely non-decreasing stochastic processes on $[0, \infty)$ governing the time-evolution of the random environment. We henceforth write $\mathbb{P}$ for the probability measure associated with the environment and let $\Lambda_{e}([a, b]):=$ $\Lambda_{e}(b)-\Lambda_{e}(a)$ be the increment of $\Lambda_{e}$ over an interval $[a, b] \subset[0, \infty)$.

We assume that for some $\zeta>9$ the following condition holds. For any $\varepsilon>0$ and any interval $I \subset[0, \infty)$ of length $|I|$,

$$
\sup _{e \in E} \mathbb{P}\left(\left|\Lambda_{e}(I)-\right| I||>|I|^{1 / 2+\varepsilon}\right) \leqslant c|I|^{-\zeta},
$$

(DIFF)

where $c=c(\varepsilon)$ is a finite constant only depending on $\varepsilon$. In particular, an application of the Borel-Cantelli lemma yields that for every $e \in E$ almost surely $\Lambda_{e}(t) / t \rightarrow 1$ as $t \rightarrow \infty$.

Given $\left\{\Lambda_{e}\right\}_{e \in E}$, we define a nearest-neighbor random walk $\{X(t)\}_{t \geqslant 1}$ on $\mathbb{Z}$ such that a walker sitting at $X(t)=v$ at time $t$ jumps along an incident edge $e$ at rate $\Lambda_{e}(t) / t$. To be more precise, the definition relies on a graphical representation. To that end, let $\left\{A_{t}(v, e)\right\}_{t \geqslant 0}$ denote a Poisson point process of arrows directed from each node $v \in V$ along an incident $e$ with intensity measure $t^{-1} \Lambda_{e}(t) \mathrm{d} t$. Now, the walker $X(t)$ is located at $X(t)=0$ for $t \in[0,1]$ and afterwards always follow the arrows. We call $X(t)$ a capricious random walk (CRW) and illustrate its transition dynamics in Figure 1. We write $\mathbf{P}_{\lambda}$ for the quenched probability measure, that is the probability measure of the walker conditioned on a particular realization $\lambda$ of the environment. Note that a priori it is not clear that there is no explosion, i.e., that the walker does not jump infinitely often in a bounded time interval. This will be excluded in Proposition 3.1 below.

Before stating the main result, we illustrate that condition (DIFF) holds for environments induced by renewal processes.

Example 2.1 (Renewal process). Let $\left\{Y_{n}\right\}_{n} \geqslant 1$ be a sequence of non-atomic iid positive random variables with $\mathbb{E}\left[Y_{1}\right]=1$ and admitting some finite exponential moment. Let $\left\{\Lambda_{e}(t)\right\}_{t \geqslant 0}$ be the associated stationary renewal process [2, Section 5.3]. Specifically, let 


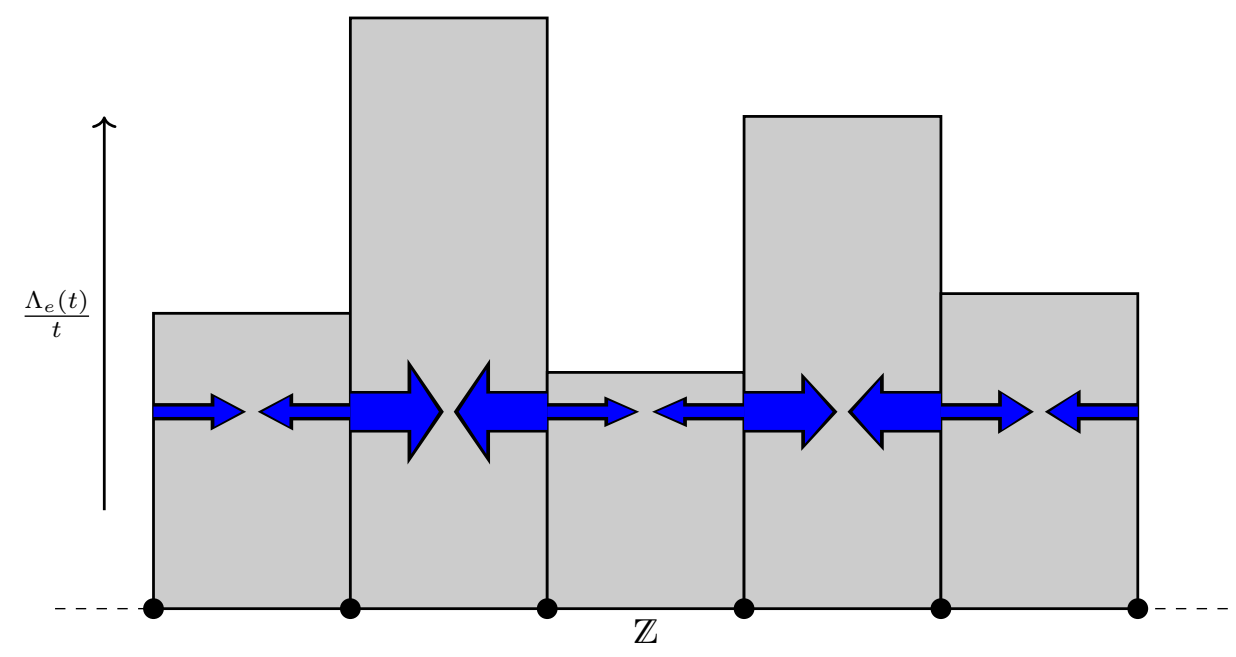

Figure 1: Transition scheme of the CRW. Arrow thickness represents jump rate towards edge.

$Y_{0}^{\prime}$ be independent of $\left\{Y_{n}\right\}_{n \geqslant 1}$ and distributed according to the size-biased distribution of $Y_{1}$, and set $Y_{0}=U Y_{0}^{\prime}$ where $U \sim \operatorname{Unif}([0,1])$ is an independent uniform random variable. Then, define the renewal process

$$
\Lambda_{e}(t):=\inf \left\{n \geqslant 0: S_{n}>t\right\},
$$

where $S_{n}:=\sum_{0 \leqslant i \leqslant n} Y_{i}$. Although $\left\{\Lambda_{e}(t)\right\}_{t \geqslant 0}$ has stationary increments by [2, Theorem 3.3], the conductance $\Lambda_{e}(t) / t$ is in general neither stationary nor Markovian.

Since the increments are stationary, it suffices to verify (DIFF) for intervals of the form $I=[0, t]$. To that end, put $n_{-}(t):=\left\lfloor t-t^{1 / 2+\varepsilon}\right\rfloor$ and $n_{+}(t):=\left\lceil t+t^{1 / 2+\varepsilon}\right\rceil$. Then,

$$
\mathbb{P}\left(\left|\Lambda_{e}(t)-t\right| \geqslant t^{1 / 2+\varepsilon}\right)=\mathbb{P}\left(\Lambda_{e}(t) \leqslant n_{-}(t)\right)+\mathbb{P}\left(\Lambda_{e}(t) \geqslant n_{+}(t)\right),
$$

and we explain how to deal with the first expression, noting that the arguments for the second are similar but easier. Now,

$\mathbb{P}\left(\Lambda_{e}(t) \leqslant n_{-}(t)\right)=\mathbb{P}\left(S_{n_{-}(t)} \geqslant t\right) \leqslant \mathbb{P}\left(Y_{0} \geqslant n_{-}(t)\right)+\mathbb{P}\left(\left(S_{n_{-}(t)}-Y_{0}\right)-n_{-}(t) \geqslant t-2 n_{-}(t)\right)$.

First, since $Y_{1}$, and therefore $Y_{0}$, is assumed to have some exponential moment, the first expression decays stretched exponentially in $t$. Moreover, since $S_{n_{-}(t)}-Y_{0}$ is a sum of $n_{-}(t)$ iid mean-1 random variables, moderate deviations theory also implies that the second probability decays stretched exponentially in $t$. In particular, condition (DIFF) is satisfied for any $\zeta>0$.

Further examples can be constructed using general theory on concentration inequalities, e.g. $[13,17]$. For instance, if $\left(\xi_{t}\right)$ is an interacting particle systems within the conemixing class [4] the process $\Lambda_{e}(t)=\int_{0}^{t} f\left(\xi_{s}(x)\right) d s$ for $e=(x, x+1)$ and $f: A^{\mathbb{Z}} \rightarrow[0, \infty)$ is a summable $\phi$-mixing process and [17, Corollary 4] applies. By [5, Theorem 2.4], this approach also includes the supercritical contact process, extending our setting beyond the cone-mixing environments. Although condition (DIFF) does not a priori impose any constraints on mixing in space and time, it is stronger than it might appear at first sight as it concerns arbitrary intervals. For instance, thinking of first-passage percolation on $\mathbb{Z}^{2}$ and defining $\Lambda_{\{i, i+1\}}(t)$ as the first-passage time to a node $(t, i)$, condition (DIFF) holds for intervals of the form $I=[0, t]$. However, it seems questionable, whether this remains true for general intervals. 
Theorem 2.2 (Quenched invariance principle). Assume that (DIFF) holds with $\zeta>9$. Then, for $\mathbb{P}$-almost every realization $\lambda$ of the environment $\left\{\Lambda_{e}\right\}_{e \in E}$, the $C R W$ in $\lambda$ satisfies the invariance principle with respect to $\mathbf{P}_{\lambda}$. That is, $\{X(t T) / \sqrt{2 T}\}_{t \leqslant 1}$ converges in distribution under $\mathbf{P}_{\lambda}$ to standard Brownian motion as $T \rightarrow \infty$.

Although of less practical value, in the mathematical literature there is also interest in the behavior of the RW under the annealed law, i.e., the joint distribution of the environment together with that of the walker. Since the quenched invariance principle implies the annealed one, Theorem 2.2 holds in the annealed setting too. In fact, by a slight adaptation of our proof, the annealed invariance principle can be shown to hold assuming $\zeta>5$ only. In either case, we do not claim the concentration exponents to be optimal. However, we could well imagine that a pathologically slow concentration could lead to a significantly slowed down walker showing up as a time-scaled BM, or even lead to a transient walker.

The main tool to prove Theorem 2.2 is a finely adapted coupling with a continuous time symmetric simple random walk (SRW), as detailed in the next section.

\section{Proofs}

For the proof of Theorem 2.2, we first formulate three central auxiliary statements in Section 3.1 and show how they imply the invariance principle, then give detailed proofs of the auxiliary results in Sections 3.2-3.6.

\subsection{Proof of Theorem 2.2}

We consider the total deviations of the CRW from the origin separately on different time scales. To make this precise, let

$$
R_{t_{1}, t_{2}}:=\sup _{t_{1} \leqslant t \leqslant t_{2}}\left|X(t)-X\left(t_{1}\right)\right|, \quad t_{2} \geqslant t_{1},
$$

denote the range of the walker in the time window $\left[t_{1}, t_{2}\right]$ and set $R_{t}:=R_{1, t}$. Sometimes, in the literature, the range is defined by taking the supremum of $\left|X(t)-X\left(t^{\prime}\right)\right|$ over pairs $t, t^{\prime} \in\left[t_{1}, t_{2}\right]$, but the two variants differ only by a factor that is irrelevant for the following.

To lead up to the proof of Theorem 2.2, we show that the range is essentially diffusive. This is manifested in the following two propositions whose proofs are postponed to Sections 3.2 and 3.6.

In the following, for a family of events $\left\{E_{T}\right\}_{T \geqslant 1}$, we say that these events occur with high probability (whp) if $\lim _{T \rightarrow \infty} \mathbf{P}_{\lambda}\left(E_{T}\right)=1$ holds for $\mathbb{P}$-almost every realization $\lambda$ of $\left\{\Lambda_{e}\right\}_{e}$. Moreover, if $\{S(T)\}_{T \geqslant 1}$ is a family of random variables and $f:[1, \infty) \rightarrow[0, \infty)$ is any function, we write $S(T) \in o_{\mathbf{P}}(f(T))$ if $S(T) / f(T)$ tends to 0 in probability with respect to $\mathbf{P}_{\lambda}$ as $T \rightarrow \infty$.

Proposition 3.1 (Linear displacement bound). The events $\left\{R_{T} \leqslant 2 T\right\}_{T \geqslant 1}$ occur whp.

Proposition 3.2 (Small displacement at intermediate times). Let $(\zeta-1)^{-1}<\alpha<\beta \leqslant 1$. Then, for every $\varepsilon>0$, for $\mathbb{P}$-almost every realization $\lambda$ of $\left\{\Lambda_{e}\right\}_{e}$,

$$
R_{T^{\alpha}, T^{\beta}} \in o_{\mathbf{P}}\left(T^{\beta-\alpha / 2+\varepsilon}\right) .
$$

If, moreover, $\beta<1$, then

$$
R_{T^{\alpha}, T^{\beta}} \in o_{\mathbf{P}}(\sqrt{T}) .
$$

We infer Proposition 3.1 from a fairly rough estimate showing that the maximal number of jumps of $X$ grows at most linearly. The key ingredient to establish Proposition 
3.2 and to conclude the proof of Theorem 2.2 is a coupling of $\{X(t)\}_{t \leqslant T}$ with a continuoustime SRW $\left\{X^{\mathrm{s}}(t)\right\}_{t \leqslant T}$ on $\mathbb{Z}$, that we discuss in more detail in Section 3.3. The coupling is initiated at time $T^{\beta}$, in position $X^{\mathrm{s}}\left(T^{\beta}\right):=X\left(T^{\beta}\right)$ for some fixed $\beta<1$ to be specified below and $X^{\mathrm{s}}$ jumps at a slightly diminished rate of $2\left(1-\varepsilon_{T}\right)$, where $\varepsilon_{T}=\varepsilon_{T}(\gamma)$ decays as $T^{-\gamma}$, for some $\gamma<1 / 2$.

Proposition 3.3 (Coupling to SRW). For some $\beta<1$ and $\gamma<1 / 2$, for $\mathbb{P}$-almost every realization $\lambda$ of $\left\{\Lambda_{e}\right\}_{e}$, there is a coupling between $\{X(t)\}_{T^{\beta} \leqslant t \leqslant T}$ and $\left\{X^{\mathrm{s}}(t)\right\}_{T^{\beta} \leqslant t \leqslant T}$ such that, for the coupled version,

$$
\sup _{T^{\beta} \leqslant t \leqslant T}\left|X(t)-X^{\mathbf{s}}(t)\right| \in o_{\mathbf{P}}(\sqrt{T}) .
$$

The invariance principle can now be deduced from Propositions 3.1-3.3.

Proof of Theorem 2.2. Let $\beta$ and $\gamma$ be as in Proposition 3.3 and let $\lambda$ be a realization of $\left\{\Lambda_{e}\right\}_{e}$ such that the statements of Propositions 3.1-3.3 hold. By extending the coupling in Proposition 3.3, we decompose $X(t)$ as $X(t)=(X(t)-Y(t))+Y(t)$, where for $t \leqslant T^{\beta}$, we let $Y(t)$ be a rate- 2 continuous time SRW independent of $X$ and $X^{\mathrm{s}}$, whereas for $t \geqslant T^{\beta}$, we set

$$
Y(t):=X^{\mathrm{s}}\left(\left(t-T^{\beta}\right) /\left(1-\varepsilon_{T}\right)+T^{\beta}\right)+\left(Y\left(T^{\beta}\right)-X^{\mathrm{s}}\left(T^{\beta}\right)\right) .
$$

In particular, $\{Y(t)\}_{t \leqslant T}$ is a rate- 2 continuous time SRW, hence we may apply the standard invariance principle to deduce that $\{Y(t)\}_{t \leqslant T}$ converges to Brownian motion in distribution under diffusive rescaling. To complete the argument, it thus suffices to show that

$$
\sup _{t \leqslant T}|X(t)-Y(t)| \in o_{\mathbf{P}}(\sqrt{T}) .
$$

To begin with, set $\alpha \in\left((\zeta-1)^{-1}, \frac{1}{2}\right)$ and note that $\sup _{t \leqslant T^{\alpha}}|X(t)| \in o_{\mathbf{P}}(\sqrt{T})$ by Propositions 3.1. Then, since $\beta<1$, by Proposition 3.2 also $R_{T^{\alpha}, T^{\beta}} \in o_{\mathbf{P}}(\sqrt{T})$. Now,

$$
\sup _{t \leqslant T^{\beta}}|X(t)| \leqslant 2 \sup _{t \leqslant T^{\alpha}}|X(t)|+\sup _{T^{\alpha} \leqslant t \leqslant T^{\beta}}\left|X(t)-X\left(T^{\alpha}\right)\right|=2 R_{T^{\alpha}}+R_{T^{\alpha}, T^{\beta}},
$$

shows that $\sup _{t \leqslant T^{\beta}}|X(t)| \in o_{\mathbf{P}}(\sqrt{T})$, and a.s. this is also the case if $X$ is replaced by $Y$. Therefore, it remains to control the deviation of $X(t)$ and $Y(t)$ for $t \geqslant T^{\beta}$.

First, by Proposition 3.3, $\left\{T^{-1 / 2}\left(X(t)-X^{\mathrm{s}}(t)\right)\right\}_{t \in\left[T^{\beta}, T\right]}$ vanishes in probability with respect to the sup-norm. Hence, it remains to show that

$$
\sup _{T^{\beta} \leqslant t \leqslant T}\left|X^{\mathbf{s}}(t)-Y^{\prime}(t)\right| \in o_{\mathbf{P}}(\sqrt{T}),
$$

with $Y^{\prime}(t)=X^{\mathrm{s}}\left(\left(t-T^{\beta}\right) /\left(1-\varepsilon_{T}\right)+T^{\beta}\right)$. To this end, we discretize and use the Markov property to obtain that

$$
\begin{aligned}
\mathbf{P}_{\lambda}\left(\sup _{T^{\beta} \leqslant t \leqslant T}\left|X^{\mathbf{s}}(t)-Y^{\prime}(t)\right|>\delta \sqrt{T}\right) & \leqslant \sum_{i \leqslant T} 2 \mathbf{P}_{\lambda}\left(2 \sup _{t \leqslant 2 T \varepsilon_{T}}\left|X^{\mathbf{s}}\left(t+T^{\beta}+i\right)-X^{\mathbf{s}}\left(T^{\beta}+i\right)\right|>\delta \sqrt{T}\right) \\
& \leqslant 2 T \mathbf{P}_{\lambda}\left(2 \sup _{t \leqslant 2 T \varepsilon_{T}}\left|X^{\mathbf{s}}\left(t+T^{\beta}\right)-X^{\mathbf{s}}\left(T^{\beta}\right)\right|>\delta \sqrt{T}\right) .
\end{aligned}
$$

Now, it follows from Doob's $L^{p}$-inequality that for $p \geqslant 2$,

$$
\mathbf{P}_{\lambda}\left(\sup _{t \leqslant 2 T \varepsilon_{T}}\left|X^{\mathbf{s}}\left(t+T^{\beta}\right)-X^{\mathbf{s}}\left(T^{\beta}\right)\right|>\delta \sqrt{T}\right) \leqslant(2 / \delta)^{p} T^{-p / 2} \mathbf{E}_{\lambda}\left[\left|X^{\mathbf{s}}\left(2 T \varepsilon_{T}+T^{\beta}\right)-X^{\mathbf{s}}\left(T^{\beta}\right)\right|^{p}\right]
$$

which is of order $O\left(\varepsilon_{T}^{p / 2}\right)$, since for the SRW $X^{\mathrm{s}}\left(t+T^{\beta}\right)-X^{\mathrm{s}}\left(T^{\beta}\right)$ and any $p>1, \mathbf{E}_{\lambda}\left[\mid X^{\mathrm{s}}(t+\right.$ $\left.\left.T^{\beta}\right)-\left.X^{s}\left(T^{\beta}\right)\right|^{p}\right] \in O\left(t^{p / 2}\right)$. Hence, choosing $p$ large enough so that $\varepsilon_{T}^{p / 2} \in o\left(T^{-1}\right)$ concludes the proof.

The remainder of the paper is devoted to the proof of Propositions 3.1-3.3. 


\subsection{Proof of Proposition 3.1}

The main idea for proving Proposition 3.1 consists of two steps. First, in Lemma 3.4 we leverage condition (DIFF) to establish a linear growth of the environment. Then, we invoke a Poisson concentration result to deduce that in this environment, the walker travels at most at linear speed.

Lemma 3.4 (Uniform boundedness of jump rates). Let $\varepsilon>0$. Then,

$$
\sup _{e \in E} \mathbb{P}\left(\sup _{s \geqslant t}\left|\Lambda_{e}(s)-s\right| s^{-\frac{1}{2}-\varepsilon}>1\right) \in O\left(t^{-(\zeta-1)}\right) .
$$

Proof. Put $\delta(s)=s^{-\frac{1}{2}+\varepsilon}$. First, for any $t>0$,

$$
\begin{aligned}
\mathbb{P}\left(\sup _{s \geqslant t}\left(\Lambda_{e}(s)-s\right) s^{-1} \delta(s)^{-1}>1\right) & \leqslant \sum_{j \geqslant t} \mathbb{P}\left(\sup _{s \in[j-1, j)}\left(\Lambda_{e}(s)-s\right) s^{-1} \delta(s)^{-1}>1\right) \\
& \leqslant \sum_{j \geqslant t} \mathbb{P}\left(\Lambda_{e}(j)-j>j \delta(j-1)-(1+\delta(j-1))\right) .
\end{aligned}
$$

We have $j \delta(j-1)-(1+\delta(j-1))>j^{(1+\varepsilon) / 2}$ for $j$ sufficiently large. From (DIFF), it thus follows, that $\mathbb{P}\left(\Lambda_{e}(j)-j>j \delta(j-1)-(1+\delta(j-1))\right) \leqslant c j^{-\zeta}$ for all sufficiently large $j$ and therefore we can find $T>1$ such that, for all $t \geqslant T$,

$$
\mathbb{P}\left(\sup _{s \geqslant t}\left(\Lambda_{e}(s)-s\right) s^{-1} \delta(s)^{-1}>1\right) \leqslant c \sum_{j \geqslant t} j^{-\zeta} \in O\left(t^{-(\zeta-1)}\right) .
$$

We conclude the proof by noting that bounds on the lower deviation of $\Lambda_{e}(s)$ can be derived in a similar manner.

We recall from [14, Lemma 1.2] a standard result on concentration of Poissonian random variables for ease of reference.

Lemma 3.5 (Poisson concentration). Let $Z$ be a Poisson random variable with parameter $\lambda>0$. Then, for all $x \geqslant \mathrm{e}^{2} \lambda$,

$$
\mathbb{P}(Z \geqslant x) \leqslant \mathrm{e}^{-\frac{x}{2} \log (x / \lambda)} .
$$

Now, we have collected all ingredients for the proof of Proposition 3.1.

Proof of Proposition 3.1. Let $\alpha \in(2 /(\zeta-1), 1 / 2)$. We consider the range of $X$ on the time intervals $\left[0, t^{\alpha}\right]$ and $\left[t^{\alpha}, t\right]$ separately. For the early times let

$$
B_{t}:=\left\{\max _{x:|x| \leqslant t} \Lambda_{\{x, x+1\}}\left(t^{\alpha}\right) \leqslant 2 t^{\alpha}\right\}, \quad t \geqslant 1,
$$

be the event that until time $t^{\alpha}$ all edges at distance at most $t$ from the origin have weight at most $2 t^{\alpha}$. From assumption (DIFF) and the Borel-Cantelli lemma, it follows that for $\mathbb{P}$-almost every realization $\lambda$, the event $B_{t}$ occurs for all sufficiently large $t$. Moreover, on the event $B_{t}$, the range process $R_{t^{\alpha}}$ is stochastically dominated by a Poisson process with intensity $2 t^{\alpha}$ on $\left[0, t^{\alpha}\right]$, unless $X$ (and therefore also the dominating Poisson process) leaves the set $[-t, t]$. Thus, by Lemma 3.5,

$$
\mathbf{P}_{\lambda}\left(R_{t^{\alpha}}>3 t^{2 \alpha} \text { or there is explosion before } t^{\alpha}\right) \leqslant \exp \left(-\frac{3}{2} t^{2 \alpha} \log \left(\frac{3}{2}\right)\right) \text { for all } \lambda \in B_{t} .
$$

Hence, by the Borel-Cantelli Lemma there is no explosion and $\lim _{t \rightarrow \infty} \mathbf{P}_{\lambda}\left(R_{t^{\alpha}} \leqslant 3 t^{\alpha}\right)=1$.

Turning to the times in the interval $\left[t^{\alpha}, t\right]$, we argue similarly: let now

$$
C_{t}:=\left\{\sup _{\substack{t^{\alpha} \leqslant s \leqslant t \\ x:|x| \leqslant 2 t}} \Lambda_{\{x, x+1\}}(s) / s \leqslant 4 / 3\right\}, \quad t \geqslant 1,
$$


be the event that, on all edges at distance at most $2 t$ from the origin and all times $s \in\left[t^{\alpha}, t\right]$, the normalized weight $\Lambda_{e}(s) / s$ is bounded above by $4 / 3$. By Lemma 3.4, for $\mathbb{P}$-almost every realization of $\lambda$ the event $C_{t}$ occurs for all sufficiently large $t$. On the event $B_{t} \cap C_{t}$, the range $R_{t^{\alpha}, t}$ is bounded above by a Poisson process with intensity $4 / 3$ on the interval $\left[t^{\alpha}, t\right]$ at least unless the dominating Poisson process jumps above $2 t$. Thus, by Lemma 3.5 with $x=\frac{3}{2} t$ and $\lambda=\frac{4}{3} t$,

$$
\mathbf{P}_{\lambda}\left(R_{t^{\alpha}, t}>2 t\right) \leqslant \exp \left(-\frac{3}{4} t \log \left(\frac{9}{8}\right)\right) \text { for all } \omega \in B_{t} \cap C_{t} .
$$

In particular, for P-almost every realization of $\lambda$, we have $\lim _{t \rightarrow \infty} \mathbf{P}_{\lambda}\left(R_{t^{\alpha}, t} \leqslant 2 t\right)=1$.

\subsection{Coupling CRW and delayed SRW}

To estimate the fluctuation of $X$ more accurately, we now introduce the coupling with a SRW $X^{\text {s }}$ formally. This coupling relies on a coupling time $T^{\beta}$ and a jump delay $\varepsilon_{T}=T^{-\gamma}$ both depending on the time horizon $T$ and on $\beta>1 / 2$ and $\gamma>1 / 4$. Later, we will choose $\beta$ and $\gamma$ to be just below 1 and $1 / 2$, respectively.

We construct the coupling appearing in Proposition 3.3 and used throughout the remaining sections via the arrow process $\left\{A_{t}(v, e)\right\}_{t \geqslant T^{\beta}}$ introduced in Section 2. From these arrows, we now construct a coupled instance $\hat{X}^{\mathrm{s}}=\left\{\hat{X}^{\mathrm{s}}(t)\right\}_{t \geqslant T^{\beta}}$ of the SRW. We start at $\hat{X}^{\mathrm{s}}\left(T^{\beta}\right)=X\left(T^{\beta}\right)$. Let $\left\{\tau_{i}\right\}_{i \geqslant 1}$, denote the jump times of $X, e_{i}$ the corresponding edges traversed and construct the jumps of $\hat{X}^{\mathrm{s}}$ recursively as follows: given $\left\{\hat{X}^{\mathrm{s}}(s)\right\}_{s \leqslant \tau_{i}}$ and $\tau_{i+1}=t$, sample a uniform random variable

$$
U_{t} \sim \operatorname{Unif}\left[0, t^{-1} \Lambda_{e_{i}}(t)\right]
$$

independently of the collection of arrows $\left\{A_{t}(\cdot, \cdot)\right\}_{t}$ and of $\hat{X}^{\mathrm{s}}(s), s \leqslant \tau_{i}$. If $U_{t} \leqslant 1-\varepsilon_{T}$, then $\hat{X}^{\mathrm{s}}(t)$ moves along the arrow. If $U_{t}>1-\varepsilon_{T}$, then $\hat{X}^{\mathrm{s}}(t)$ does not move. That is, whenever $X$ encounters an arrow from $A_{t}(v, e)$, the walker $\hat{X}^{\mathrm{s}}$ decides independently whether mimicking the movement of $\hat{X}^{\mathrm{s}}(t)$ or staying put.

Henceforth, we will need good control over the deviations in the environment. Therefore, we define the events

$$
F_{T}:=\left\{\sup _{\substack{T^{\beta} \leqslant s \leqslant T \\ x:|x| \leqslant 4 T}}\left|t^{-1} \Lambda_{x, x+1}(t)-1\right|<\varepsilon_{T}\right\}, \quad T>1 .
$$

As $F_{T}$ involves at most $8 T+2$ edges, for any $\gamma<1 / 2$, we deduce from Lemma 3.4 that

$$
\mathbb{P}\left(F_{T}^{c}\right) \in O\left((8 T+2) T^{-\beta(\zeta-1)}\right), \quad T>1,
$$

so that for $\beta>2 /(\zeta-1)$ the Borel-Cantelli Lemma implies that $F_{T}$ occurs for all sufficiently large $T$.

Lemma 3.6 (Coupling lemma). Let $\beta>2 \gamma$ and $\hat{X}^{\mathrm{s}}$ be defined as above. For P-almost every realization $\lambda$ of $\left\{\Lambda_{e}\right\}_{e}$ and large enough $T$, there exists a coupling between $\left\{\hat{X}^{\mathrm{s}}(t)\right\}_{T^{\beta} \leqslant t \leqslant T}$ and the symmetric random walk $\left\{X^{\mathrm{s}}(t)\right\}_{T^{\beta} \leqslant t \leqslant T}$ starting at $X^{\mathrm{s}}\left(T^{\beta}\right)=$ $X\left(T^{\beta}\right)$ and jumping at rate $2\left(1-\varepsilon_{T}\right)$ such that whp $\hat{X}^{\mathrm{s}}(t)=X^{\mathrm{s}}(t)$ holds for all $t \in\left[T^{\beta}, T\right]$.

To enhance readability, we tacitly assume henceforth that the probability measure $\mathbf{P}_{\lambda}$ also includes the coupling.

Proof. The significance of $F_{T}$ can be seen by noting that the jumps of $X$ can be identified as a local thinning of the edge arrow processes $\left\{A_{t}(v, e)\right\}_{t \geqslant T^{\beta}}$, so we need to avoid the situation in which the local intensity of the edge arrow processes is too low to sustain the thinning. Since an independent thinning of a Poisson process is again Poisson, the 
walker $\hat{X}^{\mathrm{s}}$ moves according to the same transition law as $X^{\mathrm{s}}$, as long as it does not deviate by more than $2 T$ from its starting location. To be more precise, introduce

$$
\tau:=\inf \left\{t \geqslant T^{\beta}:\left|\hat{X}^{\mathrm{s}}-X\left(T^{\beta}\right)\right|>2 T\right\},
$$

and note that $\tau$ is a stopping time when fixing the environment $\left\{\Lambda_{e}(\cdot)\right\}_{e}$. In particular, if $\left\{X^{*, s}(t)\right\}_{t \geqslant 0}$ is a simple random walk independent of the environment $\left\{\Lambda_{e}(\cdot)\right\}_{e}$, then setting

$$
X^{\mathrm{s}}(t):= \begin{cases}\hat{X}^{\mathrm{s}}(t) & \text { if } t \in\left[T^{\beta}, \tau\right] \text { and }\left|X\left(T^{\beta}\right)\right| \leqslant 2 T, \\ \hat{X}^{\mathrm{s}}(\tau)+X^{*, \mathrm{~s}}(t-\tau) & \text { if } t \in[\tau, T] \text { and }\left|X\left(T^{\beta}\right)\right| \leqslant 2 T, \\ X\left(T^{\beta}\right)+X^{*, \mathrm{~s}}\left(t-T^{\beta}\right) & \text { if }\left|X\left(T^{\beta}\right)\right|>2 T\end{cases}
$$

indeed defines a process whose law is that of a SRW with jump rate $2\left(1-\varepsilon_{T}\right)$. Hence, it remains to show that the event $\left\{\left|X\left(T^{\beta}\right)\right| \leqslant 2 T \leqslant 2 \tau\right\}$ occurs whp. Proposition 3.1 shows that $\left|X\left(T^{\beta}\right)\right| \leqslant 2 T$ with high probability. Moreover, on $F_{T}$ the law of $\hat{X}^{\mathrm{s}}$ coincides with that of a SRW with jump rate $2\left(1-\varepsilon_{T}\right)$ until time $\tau$, so that the assertion on $\{T \leqslant \tau\}$ follows from the diffusive scaling of the SRW.

\subsection{Proof of Proposition 3.3}

Firstly, since Proposition 3.3 is a high-probability statement, by Lemma 3.6 it suffices to prove it with $\hat{X}^{\mathrm{s}}$ in place of $X^{\mathrm{s}}$. Moreover, under the coupling between the CRW and the delayed SRW introduced in Section 3.3, we obtain a convenient representation of the deviation $X(t)-\hat{X}^{\mathrm{s}}(t)$. To describe it precisely, we let $\left\{\tau_{i}\right\}_{i \geqslant 1}$ denote the jump times of the CRW after the coupling time $T^{\beta}$ of Lemma 3.6. Furthermore, we decompose the index set of these jump times as

$$
\{1,2, \ldots\}=: \mathcal{I}^{-} \cup \mathcal{I}^{+},
$$

with $\mathcal{I}^{ \pm}$corresponding to jumps to the left and to the right, respectively. For $t \in\left[T^{\beta}, T\right]$, let $\mathcal{I}^{ \pm}(t):=\left\{i \in \mathcal{I}^{ \pm}: \tau_{i} \in\left[T^{\beta}, t\right]\right\}$ denote the jumps up to time $t$. In particular,

$$
X(t)-X\left(T^{\beta}\right)=\# \mathcal{I}^{+}(t)-\# \mathcal{I}^{-}(t), \quad t \in\left[T^{\beta}, T\right]
$$

and the increments of $X(\cdot)-\hat{X}^{\mathrm{s}}(\cdot)$ correspond to a thinning of the jumps at $\left\{\tau_{i}\right\}_{i \geqslant 1}$. More precisely, the difference in position can change only when $\hat{X}^{\mathrm{s}}$ stays put at one of the jump times. That is, for $t \in\left[T^{\beta}, T\right]$,

$$
X(t)-\hat{X}^{\mathrm{s}}(t)=\#\left\{i \in \mathcal{I}^{+}(t): \hat{X}^{\mathrm{s}}\left(\tau_{i}-\right)=\hat{X}^{\mathrm{s}}\left(\tau_{i}\right)\right\}-\#\left\{j \in \mathcal{I}^{-}(t): \hat{X}^{\mathrm{s}}\left(\tau_{j}-\right)=\hat{X}^{\mathrm{s}}\left(\tau_{j}\right)\right\},
$$

To analyze (3.2), we marginalize out the randomness of the SRW. Denoting the edge traversed at time $\tau_{i}$ by $e_{i}$, let

$$
Q_{i}:=\mathbf{P}_{\lambda}\left(\hat{X}^{\mathrm{s}}\left(\tau_{i}-\right)=\hat{X}^{\mathrm{s}}\left(\tau_{i}\right) \mid X\right)=1-\frac{\tau_{i}-\varepsilon_{T} \tau_{i}}{\Lambda_{e_{i}}\left(\tau_{i}\right)}
$$

be the probability to stay put for the SRW conditioned on the jump information of the CRW and the environment.

Proposition 3.7 (Marginalizing the SRW). Assume $\zeta>9$. Then there exists $\rho<1 / 2$, $\beta<1$ and $\gamma<1 / 2$ such that for $\mathbb{P}$-almost every realization $\lambda$ of the environment,

$$
\sup _{t \in\left[T^{\beta}, T\right]}\left|\sum_{i \in \mathcal{I}^{+}(t)} Q_{i}-\sum_{j \in \mathcal{I}^{-}(t)} Q_{j}\right| \in o_{\mathbf{P}}\left(T^{\rho}\right) .
$$

As the proof of Proposition 3.7 is a little lengthy, we defer it to the next section. To leverage Proposition 3.7, we need another lemma giving a linear upper bound on the jump counts. 
Lemma 3.8 (Linearity of jump counts). For P-almost every realization $\lambda$ of the environment,

$$
\lim _{T \rightarrow \infty} \mathbf{P}_{\lambda}\left(\tau_{\lfloor 4 T\rfloor} \geqslant T\right)=1 .
$$

Proof. By Proposition 3.1, whp, at most $4 T+2$ edges are involved in the evolution of $X$ up to time $T$. Consequently, whp, the number of jumps of $X$ on $\left[T^{\beta}, T\right]$ is dominated by a Poisson(3T)-distributed random variable $Z_{T}$. It follows that

$$
\mathbf{P}_{\lambda}\left(\tau_{\lfloor 4 T\rfloor} \geqslant T\right) \leqslant \mathbf{P}_{\lambda}\left(Z_{T} \geqslant 4 T\right),
$$

and the latter vanishes by Poisson concentration.

Together with Lemma 3.8, Proposition 3.7 yields Proposition 3.3.

Proof of Proposition 3.3. Let $\rho, \beta$ and $\gamma$ be as in Proposition 3.7. Let $\xi_{i}:=\mathbb{1}\left\{\hat{X}^{\mathrm{s}}\left(\tau_{i}\right)=\right.$ $\left.\hat{X}^{\mathrm{s}}\left(\tau_{i}-\right)\right\}, i \geqslant 1$ be the indicator that the SRW does not follow the CRW. By the observations leading up to Equation (3.2) and Proposition 3.7, it suffices to show that

$$
\lim _{T \rightarrow \infty} \mathbf{P}_{\lambda}\left(\sup _{T^{\beta} \leqslant t \leqslant T}\left|\sum_{i \in \mathcal{I}^{ \pm}(t)}\left(\xi_{i}-Q_{i}\right)\right| \geqslant T^{\rho}\right)=0,
$$

for both $\mathcal{I}^{+}$and $\mathcal{I}^{-}$, where $\rho$ is specified in Proposition 3.7 and chosen such that $\rho>\frac{1}{2}(1-\beta \gamma)$. By symmetry, we can contend ourselves with the statement for $\mathcal{I}^{+}$. Conditionally on $X$ and $\Lambda$, the indicators $\left\{\xi_{i}\right\}_{i \geqslant 1}$, are independent $\operatorname{Bernoulli}\left(Q_{i}\right)$ random variables. Writing $Y(t)=\sum_{i \in \mathcal{I}^{+}(s)} \xi_{i}$ and $\mathbb{Q}(\cdot)=\mathbf{P}_{\lambda}(\cdot \mid X)$ for the conditional distribution, a standard concentration inequality such as [10, Theorem 3.2] yields that

$$
\mathbb{Q}\left(\left|Y(t)-\mathbb{E}_{\mathbb{Q}}[Y(t)]\right|>T^{\rho}\right) \leqslant 2 \exp \left(-\frac{T^{2 \rho}}{\mathbb{E}_{\mathbb{Q}}[Y(t)]+T^{\rho} / 2}\right), \quad T^{\beta} \leqslant t \leqslant T .
$$

Note that we may work on the event $F_{T}$ from (3.1). In particular, Proposition 3.1 implies that $\mathbb{E}_{\mathbb{Q}}[Y(s)]=\sum_{i \in \mathcal{I}^{+}(s)} Q_{i}$ and, $Q_{i} \leqslant 2 \varepsilon_{T}$ for all $i$ with $T^{\beta} \leqslant \tau_{i} \leqslant T$. By Lemma 3.8, there are at most $4 T$ jumps of $X$ on $\left[T^{\beta}, t\right]$ and hence, whp,

$$
\sum_{i \in \mathcal{I}^{+}(t)} Q_{i} \leqslant 8 T \varepsilon_{T}=8 T^{1-\gamma}
$$

Since (3.4) is a uniform bound for $t \in\left[T^{\beta}, T\right]$, it can be applied to each of the $4 T$ jumps, irrespectively of their time occurrence in $\left[T^{\beta}, T\right]$. The union bound thus gives that

$$
\begin{aligned}
\mathbf{P}_{\lambda}\left(\sup _{T^{\beta} \leqslant t \leqslant T}\left|\sum_{i \in \mathcal{I}^{+}(t)}\left(\xi_{i}-Q_{i}\right)\right| \geqslant T^{\rho}\right) \leqslant & \mathbf{P}_{\lambda}\left(\sup _{T^{\beta} \leqslant t \leqslant T}\left|\sum_{i \in \mathcal{I}^{+}(t)}\left(\xi_{i}-Q_{i}\right)\right| \geqslant T^{\rho}, \# \mathcal{I}^{+}(T) \leq 4 T\right) \\
& +\mathbf{P}_{\lambda}\left(\# \mathcal{I}^{+}(T)>4 T\right) \\
\leqslant & 8 T \exp \left(-\frac{T^{2 \rho}}{8 T^{1-\gamma}+T^{\rho} / 2}\right)+\mathbf{P}_{\lambda}\left(\# \mathcal{I}^{+}(T)>4 T\right),
\end{aligned}
$$

which vanishes as $T \rightarrow \infty$.

\subsection{Proof of Proposition 3.7}

Since the random walk cannot circumvent edges, the visits to an edge to the right of $X\left(T^{\beta}\right)$ occur in pairs of a left-to-right passage followed by a right-to-left passage (and the other way around for edges to the left of $X\left(T^{\beta}\right)$, respectively). We formalize this observation by introducing a collection of pairings $\Pi_{e} \subseteq \mathcal{I}^{+}(T) \times \mathcal{I}^{-}(T), e \in E$, where $(i, j) \in \Pi_{e}$ if $e_{i}=e$ and

$$
j=\inf \left\{k>i: e_{k}=e, \tau_{k} \leqslant T\right\}
$$


is the first index after $i$ where the edge $e$ is revisited before time $T$. It may happen, for each edge $e$, that at any time $t \in\left[T^{\beta}, T\right]$ at most two indices $j$ with $e_{j}=e$ stay unpaired. Hence, we can decompose the difference of the jump probabilities according to the visited edges:

$$
\left|\sum_{i \in \mathcal{I}^{+}(t)} Q_{i}-\sum_{j \in \mathcal{I}^{-}(t)} Q_{j}\right|=\left|\sum_{e}\left(\sum_{\substack{i \in \mathcal{I}^{+}(t) \\ e_{i}=e}} Q_{i}-\sum_{\substack{j \in \mathcal{I}^{-}(t) \\ e_{j}=e}} Q_{j}\right)\right| \leqslant 4 R_{T^{\beta}, T} \max _{i \in \mathcal{I}^{ \pm}(T)}\left|Q_{i}\right|+\sum_{e \in E} B_{e}
$$

where

$$
B_{e}:=\sum_{(i, j) \in \Pi_{e}}\left|Q_{i}-Q_{j}\right|
$$

denotes an upper bound on the bias for edge $e$ accumulated until time $t$ from the paired indices. Since the random walk visits at most $2 R_{T^{\beta}, T}$ edges, the term $4 R_{T^{\beta}, T} \max _{i \in \mathcal{I}^{ \pm}(T)}\left|Q_{i}\right|$ is an upper bound on the contributions from the indices that stay unpaired. Moreover, fix $\theta:=\frac{5}{8}-\frac{1}{2(\zeta-1)}$ and decompose the edge set $E$ into the sets

$$
\begin{aligned}
& E_{\mathrm{b}}:=\left\{e \in E: \#\left\{i \in \mathcal{I}^{ \pm}(T): e_{i}=e\right\}>T^{\theta}\right\} \text { and } \\
& E_{\mathrm{c}}:=\left\{e \in E: 1 \leqslant \#\left\{i \in \mathcal{I}^{ \pm}(T): e_{i}=e\right\} \leqslant T^{\theta}\right\}
\end{aligned}
$$

of busy edges visited more than $T^{\theta}$ times and calm edges visited at most $T^{\theta}$ times, respectively.

To prove Proposition 3.7, we establish upper bounds for the per-edge bias in $E_{\mathrm{c}}$ and $E_{\mathrm{b}}$ separately.

Lemma 3.9 (Bias at busy and calm edges). Let $\varepsilon>0$ and $\gamma \in(3 / 8,1 / 2)$. Then there is $\beta_{0} \in(0,1)$ such that, for all $\beta>\beta_{0}$ and for $\mathbb{P}$-almost every realization $\lambda$ of $\left\{\Lambda_{e}\right\}_{e}$,

(i) $\max _{i \in \mathcal{I}^{ \pm}(T)} Q_{i} \in o_{\mathbf{P}}\left(T^{\frac{5}{8}-\beta+\varepsilon}\right)$.

(ii) $\max _{e \in E_{\mathrm{b}}} B_{e} \in o_{\mathbf{P}}\left(T^{1-\beta+\varepsilon}\right)$.

(iii) $\max _{e \in E_{\mathrm{c}}} B_{e} \in o_{\mathbf{P}}\left(T^{\frac{7}{8}+\frac{1}{2(\zeta-1)}-\beta+\varepsilon}\right)$.

Before establishing the lemma, we show how to conclude the proof of the proposition.

Proof of Proposition 3.7. First, by (3.5),

$\mathbf{P}_{\lambda}\left(\sup _{t \leqslant T}\left|\sum_{i \in \mathcal{I}^{+}(t)} Q_{i}-\sum_{j \in \mathcal{I}^{-}(t)} Q_{j}\right| \geqslant T^{\rho}\right) \leqslant \mathbf{P}_{\lambda}\left(4 R_{T^{\beta}, T} \max _{i \in \mathcal{I}^{ \pm}(T)}\left|Q_{i}\right| \geqslant \frac{T^{\rho}}{2}\right)+\mathbf{P}_{\lambda}\left(\sum_{e \in E} B_{e} \geqslant \frac{T^{\rho}}{2}\right)$.

To deal with the first summand, by Proposition 3.2, for any $\varepsilon>0$ we may assume that $8 R_{T^{\beta}, T} \leqslant T^{1-\beta / 2+\varepsilon}$. Under this event,

$$
\mathbf{P}_{\lambda}\left(4 R_{T^{\beta}, T} \max _{i \in \mathcal{I}^{ \pm}(T)}\left|Q_{i}\right| \geqslant T^{\rho} / 2\right) \leqslant \mathbf{P}_{\lambda}\left(\max _{i \in \mathcal{I}^{ \pm}(T)}\left|Q_{i}\right| \geqslant T^{\rho-1+\beta / 2-\varepsilon}\right)
$$

which by part (i) of Lemma 3.9 tends 0 provided that $\rho>5 / 8-\beta+\varepsilon+(1-\beta / 2+\varepsilon)=$ $13 / 8-3 \beta / 2+2 \varepsilon$. Particularly, for $\beta$ close to 1 and $\varepsilon$ close to 0 , this requirement is met for some $\rho<1 / 2$.

It remains to consider $\mathbf{P}_{\lambda}\left(\sum_{e \in E} B_{e} \geqslant T^{\rho} / 2\right)$. To that end, let

$$
F_{\mathrm{b}}:=\left\{\max _{e \in E_{\mathrm{b}}} B_{e} \leqslant T^{1-\beta+\varepsilon}\right\} \quad \text { and } \quad F_{\mathrm{c}}:=\left\{\max _{e \in E_{\mathrm{c}}} B_{e} \leqslant T^{\frac{7}{8}+\frac{1}{2(\zeta-1)}-\beta+2 \varepsilon}\right\}
$$


denote the events from Lemma 3.9. First, we deal with the busy edges. By Proposition 3.1, we may assume that $E_{\mathrm{b}}$ contains at most $6 T / T^{\theta}=6 T^{1-\theta}$ edges. Thus, on the event $F_{\mathrm{b}}$,

$$
\sum_{e \in E_{\mathrm{b}}} B_{e} \leqslant 6 T^{1-\theta+1-\beta+2 \varepsilon},
$$

and we note that $2-\theta-\beta+2 \varepsilon<1 / 2$ for $\beta$ and $\varepsilon$ sufficiently close to 1 and 0 , respectively.

The argument for the calm edges is entirely analogous, we only need to derive a sharper bound for the number of calm edges than 6T. By Proposition 3.2, we may assume that there are at most $T^{1-\beta / 2}$ calm edges. Now, we calculate, that on $F_{\mathrm{c}} \cap\left\{R_{T^{\beta}, T} \leqslant\right.$ $\left.T^{1-\beta / 2+2 \varepsilon} / 2\right\}$,

$$
\sum_{e \in E_{\mathrm{c}}} B_{e} \leqslant T^{1-\frac{\beta}{2}+\frac{7}{8}+\frac{1}{2(\zeta-1)}-\beta+2 \varepsilon}=T^{\frac{15}{8}+\frac{1}{2(\zeta-1)}-\frac{3 \beta}{2}+2 \varepsilon} .
$$

Particularly, $\frac{15}{8}+\frac{1}{2(\zeta-1)}-\frac{3 \beta}{2}+\varepsilon<1 / 2$ for $\beta$ and $\varepsilon$ sufficiently close to 1 and 0 , respectively. Using the bounds in (3.6) and (3.7) thus concludes the proof.

For the proof of Lemma 3.9 we recall from (3.3) that

$$
Q_{i}=\frac{\Lambda_{e_{i}}\left(\tau_{i}\right)-\tau_{i}+\tau_{i} \varepsilon_{T}}{\Lambda_{e_{i}}\left(\tau_{i}\right)} .
$$

Proof of Lemma 3.9. For all three parts, we work on the event $F_{T}$ from (3.1). Moreover, by Proposition 3.1, we may assume that $R_{T} \leqslant 2 T$. In particular, $\Lambda_{e}(s) / s \geqslant 1 / 2$ for all $T^{\beta} \leqslant s \leqslant T$ and $e \in E_{\mathrm{b}} \cup E_{\mathrm{c}}$.

Part (i). Under $F_{T}$, we have for $\gamma \geqslant 3 / 8$, for all $i \in \mathcal{I}^{+}(T) \cup \mathcal{I}^{-}(T)$,

$$
Q_{i} \leqslant \frac{T^{5 / 8}+T \varepsilon_{T}}{T^{\beta} / 2} \leqslant 4 T^{5 / 8-\beta} .
$$

Part (ii). Note that for $(i, j) \in \Pi_{e}$,

$$
Q_{i}-Q_{j}=\left(1-\varepsilon_{T}\right) \frac{\left(\tau_{j}-\tau_{i}\right) \Lambda_{e}\left(\tau_{j}\right)-\Lambda_{e}\left(\left[\tau_{i}, \tau_{j}\right]\right) \tau_{j}}{\Lambda_{e}\left(\tau_{i}\right) \Lambda_{e}\left(\tau_{j}\right)} .
$$

Hence, under $F_{T},\left|Q_{i}-Q_{j}\right| \leqslant 2 T^{-\beta}\left(\left(\tau_{j}-\tau_{i}\right)+\Lambda_{e}\left(\left[\tau_{i}, \tau_{j}\right]\right)\right)$. Now, we conclude the proof by summing over $(i, j) \in \Pi_{e}$ and noting that $\Lambda_{e}(T) \leqslant 2 T$.

Part (iii). The analysis is more delicate for calm edges: there are many of them, so we have to show that the contribution of each vanishes quickly. For a pair $(i, j) \in \Pi_{e}$, we consider the decomposition

$$
\left(Q_{i}-Q_{j}\right)\left(1-\varepsilon_{T}\right)^{-1}=\frac{\left(\tau_{j}-\tau_{i}\right)\left(\Lambda_{e}\left(\tau_{i}\right)-\tau_{i}\right)}{\Lambda_{e}\left(\tau_{i}\right) \Lambda_{e}\left(\tau_{j}\right)}-\frac{\tau_{i}\left(\Lambda_{e}\left(\left[\tau_{i}, \tau_{j}\right]\right)-\left(\tau_{j}-\tau_{i}\right)\right)}{\Lambda_{e}\left(\tau_{i}\right) \Lambda_{e}\left(\tau_{j}\right)},
$$

and bound the two summands separately. First, under $F_{T}$,

$$
\frac{\left(\tau_{j}-\tau_{i}\right)\left|\Lambda_{e}\left(\tau_{i}\right)-\tau_{i}\right|}{\Lambda_{e}\left(\tau_{i}\right) \Lambda_{e}\left(\tau_{j}\right)} \leqslant 4 T^{5 / 8} \frac{\tau_{j}-\tau_{i}}{T^{2 \beta}},
$$

and summing over all pairs $(i, j) \in \Pi_{e}$ yields the bound $T^{13 / 8-2 \beta}$ which is smaller than $T^{-1 / 4}$ for $\beta$ close to 1 . It remains to control the second term on the right-hand side in (3.8). To bound

$$
\sum_{(i, j) \in \Pi_{e}}\left|\Lambda_{e}\left(\left[\tau_{i}, \tau_{j}\right]\right)-\left(\tau_{j}-\tau_{i}\right)\right|,
$$


we fix $\alpha:=\frac{1}{4}+\frac{1}{\zeta-1}>\frac{3}{\zeta-1}$, and argue as in Lemma 3.4 that for $\varepsilon_{0}:=1 / 16$ the BorelCantelli Lemma implies that the event

$$
\max _{e \in E_{c}} \sup _{\substack{s, s^{\prime} \leqslant T \\\left|s^{\prime}-s\right| \geqslant T^{\alpha}}}\left|s-s^{\prime}\right|^{-1 / 2-\varepsilon_{0}}\left|\Lambda_{e}\left(\left[s, s^{\prime}\right]\right)-\left(s^{\prime}-s\right)\right|<1
$$

occurs for all sufficiently large $T$. Hence, by Jensen's inequality,

$$
\sum_{\substack{(i, j) \in \Pi_{e} \\ \tau_{j}-\tau_{i} \geqslant T^{\alpha}}}\left|\Lambda_{e}\left(\left[\tau_{i}, \tau_{j}\right]\right)-\left(\tau_{j}-\tau_{i}\right)\right| \leqslant \sum_{(i, j) \in \Pi_{e}}\left(\tau_{j}-\tau_{i}\right)^{1 / 2+\varepsilon_{0}} \leqslant\left(T^{\theta}\right)^{1 / 2-\varepsilon_{0}} T^{1 / 2+\varepsilon_{0}} \leqslant T^{(\theta+1) / 2+\varepsilon_{0}} .
$$

so that the contribution is at most $T^{(\theta+1) / 2-\beta+\varepsilon} \leqslant T^{-1 / 16}$. It remains to deal with the contributions from pairs satisfying $\tau_{j}-\tau_{i} \leqslant T^{\alpha}$. Now, under (3.9) we have $\Lambda_{e}\left(\left[\tau_{i}, \tau_{j}\right]\right) \leqslant$ $2 T^{\alpha}$, so that

$$
\sum_{\substack{(i, j) \in \Pi_{e} \\ \tau_{j}-\tau_{i} \leqslant T^{\alpha}}}\left|\Lambda_{e}\left(\left[\tau_{i}, \tau_{j}\right]\right)-\left(\tau_{j}-\tau_{i}\right)\right| \leqslant \# \Pi_{e} T^{\alpha} \leqslant T^{\theta+\alpha} .
$$

Inserting the definitions of $\theta$ and $\alpha$ concludes the proof.

\subsection{Proof of Proposition 3.2}

The main idea to prove Proposition 3.2 is to start by controlling deviations until time $T^{\alpha}$ for $\alpha<1 / 2$ and then bootstrap to successively longer time-scales. To that end, we rely on a coupling with a SRW which is a slightly adapted variant of the coupling introduced in Section 3.3. Instead of starting at time $T^{\beta}$, the coupling starts already earlier, namely at time $T^{\alpha}$. Moreover, the SRW is now slowed down stronger, namely by a factor $1-\varepsilon_{T^{\alpha}}=1-T^{-\alpha \gamma}$ instead of $1-T^{-\gamma}$. A basic consequence of this change is that in contrast to the scalings chosen in Section 3.3, it is no longer necessarily the case that the SRW and the CRW deviate in at most $o_{\mathbf{P}}(\sqrt{T})$ many steps. Rather, we leverage the number of coupling failures together with the known range of the SRW in order to bound the range of the CRW.

Proof of Proposition 3.2. We prove the two assertions separately.

Part 1. By the law of the iterated logarithm, the displacement of the SRW until time $T^{\beta}$ is at most of order $C T^{\beta / 2} \log \log T \leqslant T^{\beta-\alpha / 2}$. Hence, it suffices to bound the deviation between the SRW and the CRW $\{X(t)\}_{t \leqslant T^{\beta}}$. Conditioning on $\{X(t)\}_{t \leqslant T^{\beta}}$ shows that there are whp at most $T^{\beta} \varepsilon_{T^{\alpha}}=T^{\beta-\gamma \alpha}$ coupling failures. Hence, we obtain the desired worst-case deviation since $\gamma<1 / 2$.

Part 2. Recall that $0<\alpha<\beta<1$ and, for $k \geqslant 1$, let $r_{k}=1-(1-\alpha)^{k}$. Denote by $K=K(\beta)=\inf \left\{k \geqslant 1: r_{k} \geqslant \beta\right\}$, which is well defined since $\lim _{k \rightarrow \infty} r_{k}=1$, and note that

$$
R_{T^{\alpha}, T^{\beta}} \leqslant \sum_{k \leqslant K-1} R_{T^{r_{k}, T^{r}+1}}
$$

Further, note that

$$
r_{k+1}-\frac{r_{k}}{2}=1-(1-\alpha)^{k+1}-\frac{1-(1-\alpha)^{k}}{2}=1 / 2-\varepsilon(k),
$$

where $\varepsilon(k):=(1-\alpha)^{k}(1 / 2-\alpha)>0$. Hence, by part 1 , each term on the right-hand side of (3.10) is $o_{\mathbf{P}}(\sqrt{T})$, from which we conclude the proof. 
Random walks on dynamically averaging random conductances

\section{References}

[1] S. Andres, A. Chiarini, J.-D. Deuschel, and M. Slowik. Quenched invariance principle for random walks with time-dependent ergodic degenerate weights. Ann. Probab., 46(1):302-336, 2018. MR3758732

[2] S. Asmussen. Applied Probability and Queues. Springer, New York, second edition, 2003. MR1978607

[3] L. Avena, O. Blondel, and A. Faggionato. Analysis of random walks in dynamic random environments via $L^{2}$-perturbations. Stochastic Process. Appl., 128(10):3490-3530, 2018. MR3849817

[4] L. Avena, F. den Hollander, and F. Redig. Law of large numbers for a class of random walks in dynamic random environments. Electron. J. Probab., 16:587-617, 2011. MR2786643

[5] S. Bethuelsen. On projections of the supercritical contact process: uniform mixing and cutoff phenomenon. Genealogies of interacting particle systems, Lecture Notes Series, IMS, National University of Singapore, 38:315-340, 2020.

[6] S. A. Bethuelsen and M. Heydenreich. Law of large numbers for random walks on attractive spin-flip dynamics. Stochastic Process. Appl., 127(7):2346 - 2372, 2017. MR3652417

[7] M. Birkner, J. Černý, A. Depperschmidt, and N. Gantert. Directed random walk on the backbone of an oriented percolation cluster. Electron. J. Probab., 18:no. 80, 35, 2013. MR3101646

[8] M. Biskup. An invariance principle for one-dimensional random walks among dynamical random conductances. Electron. J. Probab., 24:Paper No. 87, 29, 2019. MR4003140

[9] O. Blondel, M. R. Hilário, and A. Teixeira. Random walks on dynamical random environments with nonuniform mixing. Ann. Probab., 48(4):2014-2051, 2020. MR4124532

[10] F. Chung and L. Lu. Concentration inequalities and martingale inequalities. Inter. Math., 3:79-127, 2006. MR2283885

[11] F. den Hollander and R. S. dos Santos. Scaling of a random walk on a supercritical contact process. Ann. Inst. H. Poincaré Probab. Statist., 50(4):1276-1300, 2014. MR3269994

[12] M. Kardar, G. Parisi, and Y. Zhang. Dynamic scaling of growing interfaces. Phys. Rev. Lett., 56:889, 1986.

[13] L. Kontorovich and K. Ramanan. Concentration inequalities for dependent random variables via the martingale method. Ann. Probab., 36(6):2126-2158, 2008. MR2478678

[14] M. D. Penrose. Random Geometric Graphs. Oxford University Press, Oxford, 2003. MR1986198

[15] M. D. Penrose. Growth and roughness for ballistic deposition. J. Stat. Phys., 131(2):247-268, 2008. MR2386580

[16] F. Redig and F. Völlering. Random walks in dynamic random environments: a transference principle. Ann. Probab., 41(5):3157-3180, 2013. MR3127878

[17] P.-M. Samson. Concentration of measure inequalities for Markov chains and $\Phi$-mixing processes. Ann. Probab., 28(1):416-461, 2000. MR1756011

[18] F. Solomon. Random walks in a random environment. Ann. Probability, 3:1-31, 1975. MR0362503

Acknowledgments. The authors thank the anonymous referee for reading through the entire manuscript thoroughly and for providing us with high-quality and constructive feedback. In particular, the referee suggested the extension to the quenched invariance principle. The authors thank H. Pitters for inspiring discussions in the early phase of this project. 


\section{Electronic Journal of Probability Electronic Communications in Probability}

\section{Advantages of publishing in EJP-ECP}

- Very high standards

- Free for authors, free for readers

- Quick publication (no backlog)

- Secure publication $\left(\mathrm{LOCKSS}^{1}\right)$

- Easy interface (EJMS²)

\section{Economical model of EJP-ECP}

- Non profit, sponsored by $\mathrm{IMS}^{3}, \mathrm{BS}^{4}$, ProjectEuclid ${ }^{5}$

- Purely electronic

\section{Help keep the journal free and vigorous}

- Donate to the IMS open access fund ${ }^{6}$ (click here to donate!)

- Submit your best articles to EJP-ECP

- Choose EJP-ECP over for-profit journals

\footnotetext{
${ }^{1}$ LOCKSS: Lots of Copies Keep Stuff Safe http://www. lockss.org/

${ }^{2}$ EJMS: Electronic Journal Management System http://www.vtex.lt/en/ejms.html

${ }^{3}$ IMS: Institute of Mathematical Statistics http://www.imstat.org/

${ }^{4}$ BS: Bernoulli Society http://www. bernoulli-society.org/

${ }^{5}$ Project Euclid: https://projecteuclid.org/

${ }^{6}$ IMS Open Access Fund: http://www.imstat.org/publications/open.htm
} 